\title{
Frozen frogs spring back to life
}

The astounding ability of the wood frog (Rana sylvatica) to survive for weeks at a time with two-thirds of its body water completely frozen has caught the attention of researchers. The frogs encounter subzero temperatures as they spend the winters in relatively exposed sites on the forest floor, and they may endure multiple freeze-thaw episodes over the course of a single winter.

In a new study, Jon P. Costanzo (Miami University, Oxford, $\mathrm{OH}$ ) reports that solutes called cryoprotectants lower the freezing temperature of the frogs' body fluids (J. Exp. Biol. 216, 3461-3473; 2013). Costanzo and his team looked at the blood and tissue concentrations of several metabolites among late-summer, fall and winter Alaskan wood frogs. Compared with late-summer frogs, winter frogs had elevated plasma levels of the two major cryoprotectants, urea and glucose, as well as a greater reserve of glycogen in the liver, which could be converted to glucose during freezing.

The Alaskan wood frogs were also compared with their conspecifics from

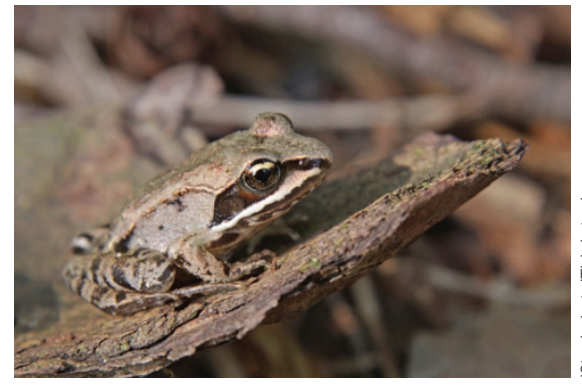

the amount of ice that forms in the body. When the freezing responses of the two wood frog populations were compared, the Alaskan frogs contained much less ice upon freezing than Ohioan frogs, owing to their higher plasma concentrations of glucose and urea.

The researchers also compared the freeze tolerances of frogs from the Alaskan population and the Ohio population. While the Ohioan wood frogs could be Ohio, a more temperate part of the species' geographic range. Plasma glucose and urea were found in much higher concentrations in the Alaskan wood frogs than in their southern counterparts. The researchers also detected the presence of an additional mystery solute in the Alaskan frogs, which they have yet to identify. The Ohioan frogs also had a 20\% lower concentration of glycogen in their livers than the Alaskan frogs.

Prolonged exposure to subzero temperatures causes the formation of ice in tissues, which dehydrates the body's cells and causes them to die. Cryoprotectants limit frozen at $-4^{\circ}$ to $-6^{\circ} \mathrm{C}$ and revived, the Alaskan wood frogs survived being frozen at temperatures below $-16^{\circ} \mathrm{C}$. These temperatures are consistent with the annual low temperatures found in the frogs' respective homes.

The ability to freeze and unfreeze living tissues while preserving viability has potentially profound implications for organ transplantation. "There's an obvious parallel between what these frogs are doing to preserve their tissues and our need to be able to cryopreserve human organs for tissue-matching purposes," Costanzo says. Kara Rosania

\section{GENOME EDITING TAKES OFF}

Genomic engineering is a staple of modern translational research; the ability to create alterations at the DNA level and explore their effects in various organisms has deepened our understanding of basic biologic function and of many human health disorders. About a year ago, scientists developed a new approach to genomic engineering called the CRISPR/Cas9 system (Science 337, 816-821; 2012). The system has since been used to engineer many biological model species, including yeast, zebrafish, mice and nematodes. Now, a trio of collaborators from the University of Wisconsin-Madison reports the successful application of this approach in fruit flies (Genetics 194, 1029-1035; 2013). Their results highlight the precision of the CRISPR/Cas9 system and show that genetic alterations made using the system are transmitted to the next generation of flies. Such precision and transmissibility are essential properties in genomic engineering.

In the CRISPR/Cas9 system, the enzyme Cas9 is directed to snip a cell's DNA at a target sequence, stimulating the cell's DNA repair machinery to fill in the break and integrate a genetic alteration of interest. The alteration can be as specific as changing a single base pair in the DNA sequence. The system uses short RNA sequences to direct DNA clipping by Cas9, whereas other genome editing approaches use proteins to direct DNA breaks. The use of short RNAs confers several advantages: they can be generated relatively rapidly, whereas generation of proteins is time-consuming and costly. Moreover, they enable broad applicability of the engineering approach across species.

Kate $0^{\prime}$ Connor-Giles, who led the fruit fly study along with Melissa Harrison and Jill Wildonger, explained in a press release, "This is so readily transferable that it's highly likely to enable gene knockout and other genome modifications in any organism. It's going to turn non-model organisms into genetic model organisms."

Wildonger described how applying the CRISPR/Cas9 system in fruit flies can result in clinical benefits. "It can be very difficult and time-consuming to generate models to study all the gene variants associated with human diseases," she said. "With this genome editing approach, if we work in collaboration with a clinician to find mutations, we can rapidly translate these into a fruit fly model to see what's happening at the cellular and molecular level."

The broad applicability and fine control offered by the CRISPR/Cas9 approach promise new insights into the fundamental workings of biological systems as well as the mechanisms underlying human disease. 